\title{
TESTBED IMPLEMENTATION OF ENERGY EFFICIENT MULTI ANTENNA SYSTEM AND ITS PERFORMANCE EVALUATION
}

\author{
Payal A. Mangale ${ }^{1}$, Y. Ravinder ${ }^{2}$ and P.S. Varade ${ }^{3}$ \\ Department of Electronics and Telecommunication Engineering, Pune Institute of Computer Technology, India
}

\begin{abstract}
With the recent development and requirement for high-speed wireless communications, the demand of energy has increased exponentially in order to support required quality of service $(Q o S)$. Multiple Input Multiple Output (MIMO) technique gaining more popularity in current communication network, since it provides advantages in terms of higher data rates and maximum capacity with the cost of more energy consumption. In this paper, QAM modulation scheme is analyzed as the best modulation scheme for the total energy required to transmit the given number of bits is reduced. A SDR testbed has been built to implement Almouti coded MIMO system using LABVIEW and Universal Software Radio Peripheral (USRP) platform. The analysis was then extended to evaluate the energy consumption of the system where it was observed that by minimizing the transmission power the energy requirement of the system gets reduced. Then the system efficiency in terms of transmit power was evaluated using USRP. It has been observed that by adjusting carrier frequency transmit power of the system gets reduced up to $25 \%$. Finally the performance of the system is evaluated by demonstrating the effect of transmission distance, symbol rate, spectral efficiency and circuit power on energy consumption of the system using LABVIEW math script.
\end{abstract}

\section{Keywords:}

Energy Efficiency, MIMO, Symbol Rate, Spectral Efficiency, USRP

\section{INTRODUCTION}

Multiple antenna system in wireless communication is evolving as the fastest technology for achieving higher capacity and data rates. This is possible through spatial multiplexing and spatial diversity techniques [1]. High energy is consumed in wireless network due to rapid increase in the high-data rate application. Therefore, energy-efficient communications, under the back-ground of limited energy resources is gaining high degree of attention. Thus, for reliable data transmission in wireless networks, energy efficient model is required. This limitation motivates the researcher to develop an energy efficient MIMO system.

Nowadays, designing an energy efficient system has gained tremendous attention in both industry and academia [2]. Since, large number of subscribers (more than 4 billion) around the world communicate through mobile phones in their day-to-day life, which unavoidably comes at the cost of increased energy consumption [3]. Most of the research is concerned in reducing the transmission energy per bit using efficient transmission schemes. In [4], some strategies for energy consumption minimization have been discussed in wide-band region. In [5], the author has proposed an optimal scheduling algorithm for minimizing the transmission energy by maximizing transmission time for buffered packets. In [6], author proposes an idea for selection of energy-efficient modulation schemes when overall energy consumed is a function of two parameters such as circuit energy and transmission energy. Some joint energy-minimizing techniques have been proposed for conventional system in [6]-[7], where optimized system parameters with multimode operation were investigated. In [7], MQAM modulation is analyzed and optimal strategies are proposed for minimization of total energy consumption for AWGN channel. Detailed tradeoff analysis between the transmission energy, the circuit energy, the transmission time and the constellation size in case of AWGN channels for coded and uncoded MQAM and MFSK is proposed. The best modulation and transmission scheme required to transmit a given number of bits for minimization of the total energy consumption is discussed in [8]. It was also observed that by co-operating among nodes MIMO systems show that for long distance communication the system efficiency is improvised in terms of energy consumption [9]. Several antenna selection strategies are employed to improve energy efficiency. Depending on the availability of large number of antennas, antenna selection strategy is employed at the transmitting end [10].Previous works on antenna selection only focused on fixed total transmit power and active RF chains. In [11] Energy efficiency can be maximized through joint optimization with the help of antenna selection strategy over the transmit power, number of active RF chains, and the antenna subset. Antenna selection is the optimal strategy with reduced complexity and low cost. Above mentioned work on energy efficiency only considers the theoretical analysis of energy efficiency in case of multiple antenna system. Thus, in this work the actual testbed implementation of multiple antenna system is being considered.

In this paper, the practical implementation of the $2 \times 2$ Almouti Coded MIMO system using LABVIEW (Laboratory Virtual Engineering Workbench) and USRP (Universal Software Radio Peripheral) is being considered. System power consumption is then evaluated by considering RF transceiver circuit blocks. The total energy consumption includes circuit energy and transmit energy. Since USRP-2922 hardware devices are used, RF circuitry is fixed and the circuit power cannot be reduced. Thus, only the transmit power is taken into consideration. The USRP transmit power is measured directly with the help of oscilloscope and current and voltage probes. Where it was observed that carrier frequency is closely related with the RF circuit power whereas baseband signal power mainly defined by symbol rate and digital logic. This power is quite small [7]. Hence, the power of baseband signal processing blocks is neglected intentionally. On the other hand, with the help of testbed it was observed that carrier frequency is directly related with transmission power. For minimizing the transmission power, the transmission time with lower carrier frequency for making energy efficient system needs to be maximized.

This paper is organized as follows. Section 2, consists of MIMO system model. In section 3, energy efficiency of the 
MIMO system is described in detail. In section 4 , the actual effects of reducing the transmit power has been verified and it is observed that around $25 \%$ power gets reduced. Simulation results are also discussed to evaluate the performance of the system in section 5 .

\section{SYSTEM MODEL}

Multiple antenna system consists of multiple antennas at the transmitter and receiver end which is used to send and receive data from multiple users on the same frequency/time channel. Transmission/reception in a multiple antenna system which involves data transmission not only between corresponding antennas but also between adjacent antennas. Basic building blocks of $2 \times 2$ MIMO system can explained with the help of following Fig.1 and Fig.2 [15].

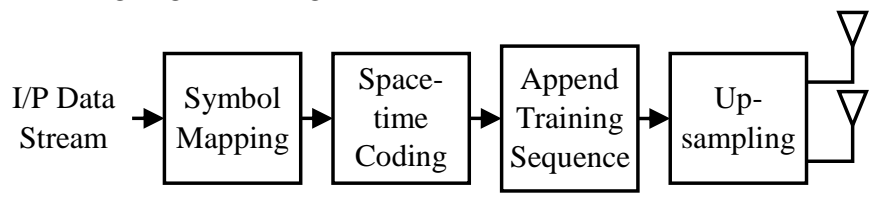

Fig.1. MIMO Transmitter

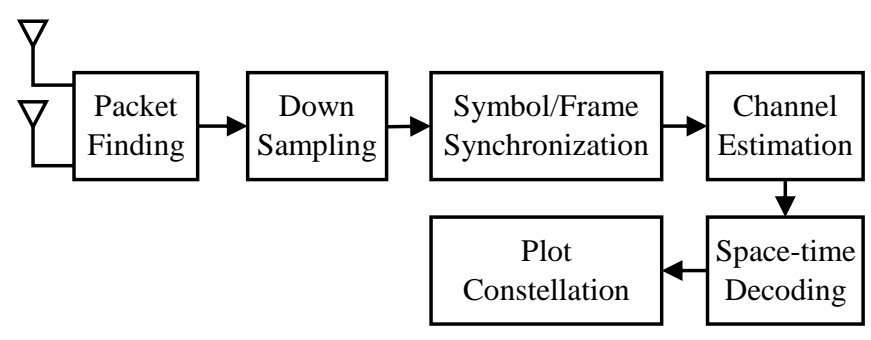

Fig.2. MIMO Receiver

Implementation of Almouti coded $2 \times 2$ MIMO system is carried out using LABVIEW simulation software. As shown in Fig.2, in transmitter section, the first step is to initialize the system parameters. This includes symbol rate, symbol per packets, modulation scheme etc. Transmitted bits are generated which acts as a message bits. With the help of suitable modulation scheme such as 4-QAM, bits can be mapped to symbols. Multiple antenna systems uses spatial diversity technique for reliable transmission of data [13]. Space time code (STC) is a technique used for reliable data transmission in wireless networks using multiple antennas. In this technique data transmission usually takes place redundantly from both ends with a hope that at least some of them can be regained in good state for reliable decoding at receiving end. Different type STCs are available but space time block codes which are also known as Almouti Code are best choice mainly because of its simple implementation. Training sequences are appended at the transmitter side which is essential for continuity of the transmitted sequence. At the receiver end training sequences are used for Synchronization and channel estimation. After appending training sequences the signal can be up-sampled and passed through pulse shaping filter which generates a sampled continuous time I/Q baseband waveform. Then the signals can be transmitted through two USRP antennas. In order to synchronize the devices for MIMO transmission the USRP had to be configured specially for that purpose. At receiving side, USRP fetches receiver data and determines the start of the packet and discard remaining non-packet portion of the frame. Process is done by comparing the value with threshold. Matched filter can be applied to determine the stream with the stronger signal. Symbol and frame synchronization is basically depends upon the two training sequences at the transmitter side. Channel estimation is done and finally the output of the system is observed using constellation graph which is explained in implementation section.

\section{ENERGY EFFICIENCY OF MIMO SYSTEM}

\subsection{POWER CONSUMPTION MODEL}

In order to quantify the energy efficiency of wireless system, a power consumption model that describes how much power is consumed is required. Hence, a proper power consumption model is important for energy efficiency analysis. This paper describes the power consumption model available for MIMO system. In this model the overall power consumption is described by the summation of power consumption of all components (i.e. signal processing blocks). In general, the total energy of the system is dependent on the two factors such as circuit energy and transmit energy. In MIMO signal processing the whole system consists of two parts which are basically RF blocks and baseband signal blocks. In this paper, the power related to the RF circuitry is considered because it is more as compared to the baseband power since baseband signal power is mainly dependent on symbol rate and the complexity of the digital logic circuit. On the other hand, RF circuit power is closely related with the carrier frequency. Based on the above assumptions, all the signal processing blocks at the transmitter and receiver are considered in the system model [9] which is also the part of the hardware, given in the following Fig.3.
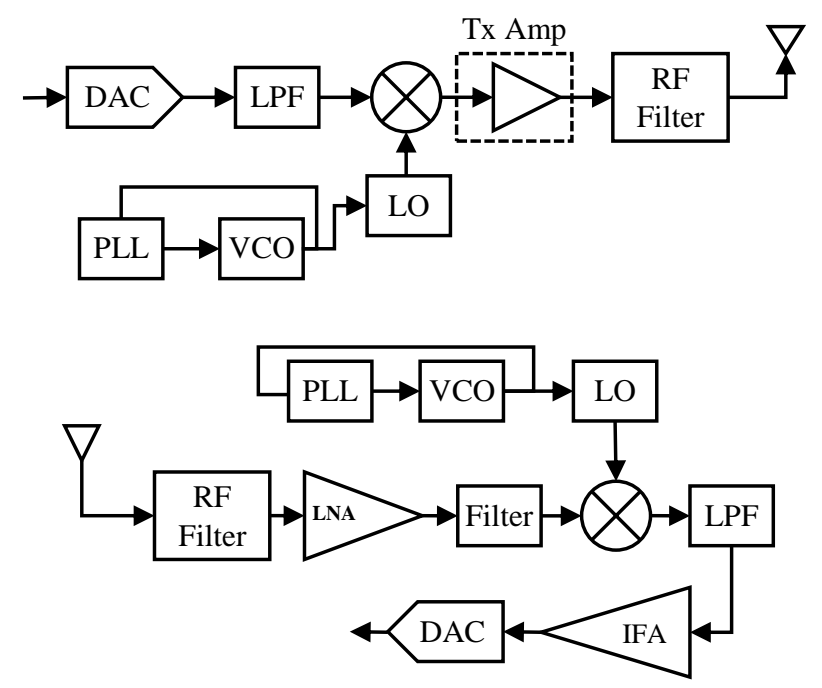

Fig.3.Transceiver circuit Blocks

The transmitter and receiver circuit paths are as shown in Fig.3, on the transmitter side host PC is used to generate the baseband signal which is then converted to an analog signal by Digital to analog converter (DAC). Later, low pass filter is used to filter and is modulated by mixer. After mixing the signal the transmission amplifier (TX amp) or power amplifier filter and amplify them and then transmit through wireless channel. Similar functional blocks can be observed on the receiver side. Low noise 
amplifier is used to filter and amplify the RF signal. The reference signal can be frequency locked with the help of device clocks and local oscillator. Mixer is used to down-convert the in-phase $(I)$ and quadrature-phase $(Q)$ components. Noise and high frequency components present in the signal are reduced by low pass filter. The $I$ and $Q$ data can be digitized with the help of analog-to-digital converter (ADC). Transceiver circuitry exhibits multimode operation. When the signal is there for transmission then the circuit works in the active mode, and when there is no signal for transmission then the overall circuit works in the sleep mode.

The overall power consumption of all the blocks on transmitter and receiver side is considered. Thus, the overall power consumption can be expressed as [9],

$$
P_{\text {Total }}=P_{P A}+P_{b b}+P_{c}
$$

where, $P_{P A}$ is the power amplifier power, $P_{b b}$ is the power of baseband signal blocks at transmitter and receiver and $P_{c}$ is the power consumed by the circuit.

Circuit power consumption is dependent on all circuit blocks used in the model which is given as,

$$
\begin{aligned}
P_{c}= & \left(P_{D A C}+P_{m i x}+P_{f i l}+P_{s y n}\right)+ \\
& \left(P_{L N A}+P_{f i l}+P_{m i x}+P_{s y n}+P_{I F A}+P_{A D C}\right)
\end{aligned}
$$

where, $P_{D A C}, P_{m i x}, P_{f i l}, P_{s y n}, P_{L N A}, P_{I F A}, P_{A D C}$ are the values of power consumption of DAC, mixer, low noise amplifier, IFA and $\mathrm{ADC}$ at transmitting and receiving side respectively. The power consumption values in Eq.(4) can be estimated using the model introduced in [6].

Table.1. QAM System Parameters

\begin{tabular}{|c|c|}
\hline Parameters & Value \\
\hline$P_{m i x}$ & $30.3 \mathrm{~mW}$ \\
\hline$P_{f i l}$ & $2.5 \mathrm{~mW}$ \\
\hline$P_{s y n}$ & $50 \mathrm{~mW}$ \\
\hline$P_{L N A}$ & $20 \mathrm{~mW}$ \\
\hline$P_{I F A}$ & $3 \mathrm{~mW}$ \\
\hline$N_{f}$ & $5-7 \mathrm{~dB}$ \\
\hline$M_{1}$ & $40 \mathrm{~dB}$ \\
\hline$N_{t x}$ & 2 \\
\hline$M_{t x}$ & 2 \\
\hline$P_{A D C}$ & $5.83 \mathrm{~mW}$ \\
\hline$P_{D A C}$ & $2.43 \mathrm{~mW}$ \\
\hline$\eta$ & 0.35 \\
\hline
\end{tabular}

The transmission energy is delivered to the antenna by the transmission amplifier. Hence, the Power of power amplifier $P_{P A}$ is a function of actual transmitted power $P_{t}$. Thus, the power consumption of the power amplifier is given by,

$$
P_{P A}=\frac{P_{t}}{\eta}
$$

where, $\eta$ is the efficiency of power amplifier.

In MIMO systems, number of signal processing blocks at transmitting and receiver side increases proportionally with number of transmit and receive chains. Thus, power consumption model in a MIMO system with $M_{t x}$ active transmit chains and $N_{t x}$ active receive chains is obtained as,

$$
\begin{gathered}
P_{\text {Total }}=\left(P_{P A}+P_{c t x}\right) M_{t x}+N_{t x} P_{c r x}+P_{b b} \\
P_{c t x}=P_{D A C}+P_{m i x}+P_{f i l}+\frac{P_{s y n}}{M_{c t x}} \\
P_{c r x}=P_{L N A}+P_{m i x}+P_{f i l}+P_{I F A}+P_{A D C}+\frac{P_{s y n}}{N_{t x}}
\end{gathered}
$$

The Eq.(7) represents the circuit power consumption per transmit branch whereas Eq.(8) represents the circuit power consumption of receive branch. Here, it is assumed that all the antenna paths share a common local oscillator. Power consumption of baseband signal blocks is intentionally omitted here. Hence, the total power consumed by the system depends on the power consumption of the power amplifier which in turn a function of transmit power. The RF circuit blocks, considered for hardware are fixed, the concentration is only on total transmit power.

\subsection{ENERGY EFFICIENCY ANALYSIS}

Generally, energy efficiency of the wireless communication system is defined in two ways as energy consumption per bit and bit per joule. These are as follows.

Energy Consumption in Bit per Joule: It is one of the most popular energy efficiency metric which is defined as the ratio of capacity to the overall power consumption. Mathematically it can be expressed as,

$$
\eta_{E E}=\frac{C}{P_{\text {Total }}}
$$

where, $\eta_{E E}$ is the energy efficiency, $C$ is the channel capacity (bits/s), $P_{\text {Total }}$ is the total power consumption of the system.

Energy Consumption per bit: In addition to above metric energy consumption is often measured in terms of energy consumption per bit. Mathematically it is given as,

$$
\eta_{E E}=\frac{P_{\text {Total }}}{R_{b}}
$$

where, $R_{b}$ is the bit rate (bps).

For given BER requirement, the actual transmit power $P_{t}$ and thus $P_{\text {Total }}$ can be computed using link budget relationship [16].

$$
P_{t}=E_{b} R_{b} \frac{(4 \pi d)^{2}}{G_{t x} G_{r x} \lambda^{2}} M_{l} N_{f}
$$

where, $E_{b}$ is the required energy per bit, $G_{t x}$ and $G_{r x}$ are the gain of transmitting and receiving antennas respectively. When the rate of the system is fixed then total energy consumption per bit can be given as,

$$
E_{b i t}=\frac{\left(P_{P A}+P_{c t x}\right)}{R_{b}}
$$

Therefore,

$$
E_{b i t}=(1+\alpha) E_{b} \frac{(4 \pi d)^{2}}{G_{t x} G_{r x} \lambda^{2}} M_{l} N_{f}+\frac{\left(P_{o u t}\right)}{R_{b}}
$$


where, $P_{\text {out }}$ is the total circuit power including circuit power $P_{c t x}$ and power amplifier power $P_{P A}$ which is nothing but the transmit power of the system. In simplified form the energy is the product of power multiplied by time hence, it can be given as,

$$
E_{b i t}=P_{t} T_{\text {on }}
$$

It can be noted that [6] the transmission energy is increases linearly with the bandwidth efficiency given as, $B_{e}=L / B T_{\text {on }}$ and $E_{t}$ decreases linearly with $T_{o n}$

Hence, the system efficiency can be achieved by selecting the proper transmission time under the given system constraints. Thus, the optimization problem can be modeled as,

$$
\begin{array}{ll}
\text { Minimize } & E_{t} \\
\text { Subject to } & 0 \leq T_{\text {on }} \leq T_{\text {Total }} \\
& 0 \leq P_{t}+P_{c t x} \leq P_{\max }
\end{array}
$$

The optimal transmission time results in optimal constellation size as $T_{o n}=L / b B$. The $E_{b}$ is defined by the target BER and underlying constellation size $b$ according to following relationship [16].

$$
P_{b} \approx \varepsilon_{H}\left\{Q \sqrt{2 \gamma_{b}}\right\}
$$

where,

$$
\begin{gathered}
\gamma_{b}=\left(\frac{\|H\| f^{2}}{M_{t x}}\right)\left(\frac{E_{b}}{N_{o}}\right) \\
E_{b} \leq \frac{2}{3}\left(\frac{P_{b}}{4}\right) \frac{-1}{M_{t x}} \frac{2^{b-1}}{\frac{1}{b^{M_{t x}}}+1} M_{l} N_{o}
\end{gathered}
$$

The overall energy consumption per bit from Eq.(13) can be given as,

$$
E_{b i t}=\frac{2}{3}\left(\frac{P_{b}}{4}\right)^{\frac{-1}{M_{t x}}} \frac{2^{b-1}}{\frac{1}{b^{M_{t x}}}+1} M_{l} N_{o} \frac{\left(4 \pi d^{2}\right)}{G_{t x} G_{r x} \lambda^{2}} M_{l} N_{f}+\frac{\left(P_{o u t} T_{o n}\right)}{L}
$$

Since $T_{o n}$ is a function of $b, E_{b i t}$ can be minimized over the variable $d \cdot b=\log _{2} M$ gives the constellation size. When $(b \geq 2)$ then second term in Eq.(18) decreases linearly with the variable $b$.

\section{MEASUREMENT IMPLEMENTATION}

\section{SETUP}

AND

An indoor wireless environment is considered for experimentation. The experimental setup in Fig.4 consists of USRP-2922 devices and LABVIEW simulation software. USRP device is used for receiving the signals present in the real time environment. Experimental setup consists of two transmitters and two receivers in which MIMO cable is used for the synchronization. Ethernet cable is used for connecting USRP devices to host PC. The Table. 2 summarizes the parameters used for the simulation of $2 \times 2$ MIMO system.

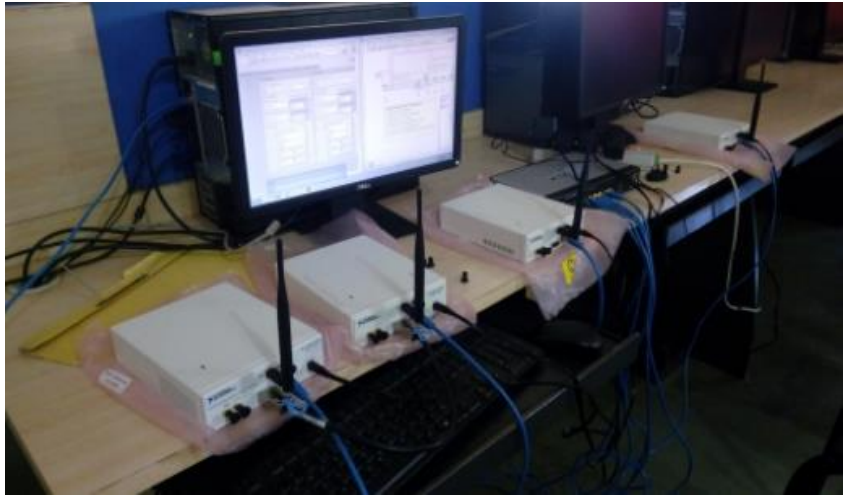

Fig.4. Actual setup for $2 \times 2$ MIMO System

Table.2. USRP Configuration Parameters

\begin{tabular}{|c|c|c|c|}
\hline Parameters & USRP Tx & USRP Rx & \\
\hline Symbol Rate (Mbps) & - & - & 1 \\
\hline Symbols & - & - & 2000 \\
\hline Oversample factor & 10 & 10 & - \\
\hline I/Q Sampling rate (S/sec) & 10 & 10 & - \\
\hline Modulation Scheme & 4-QAM & 4-QAM & - \\
\hline Carrier Frequency & $512 \mathrm{M}$ & $512 \mathrm{M}$ & - \\
\hline Active Antenna & Tx & Rx 1 & - \\
\hline
\end{tabular}

Fig.5.Constellation plot for $2 \times 2$ MIMO system

A $2 \times 2$ MIMO system in terms of constellations is visualized in Fig. 5 where we observed the two different plots. The Upper plot describes the energy spectrum of four received signals from two different signals whereas lower plot constitute of three different plots such as left most plot represents receiver plot for antenna 1 , middle one represents receiver plot for antenna 2 and right most plot represents original signal retrieved with the help of both receiver plots. 


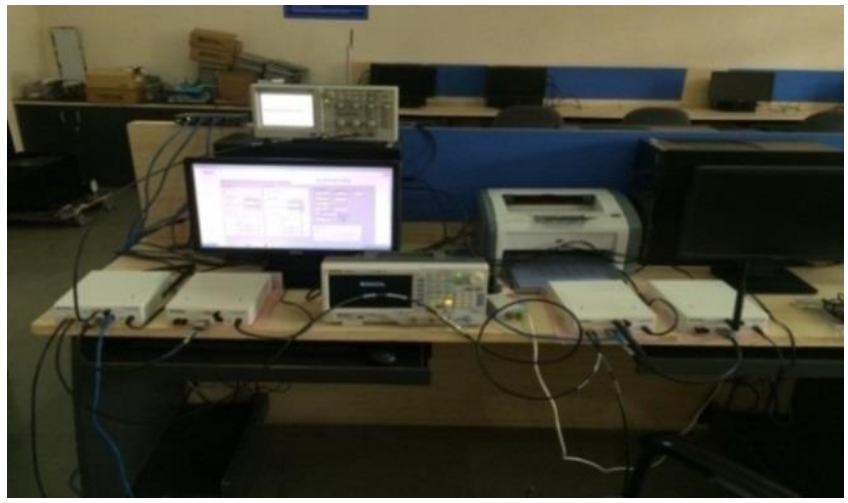

Fig.6. Actual setup for measurement of Transmit power

Table.3. USRP Transmit Power Calculations

\begin{tabular}{|c|c|c|c|c|c|c|}
\hline \multirow{2}{*}{$\begin{array}{c}\text { Carrier } \\
\text { Frequency } \\
\text { (MHz) }\end{array}$} & \multicolumn{3}{|c|}{$\mathbf{T} \mathbf{x}_{1}$} & \multicolumn{3}{|c|}{$\mathbf{T} \mathbf{x}_{2}$} \\
\hline & $\begin{array}{c}\text { Voltage } \\
(\mathrm{mV})\end{array}$ & $\begin{array}{c}\text { Current } \\
(\mathbf{m A})\end{array}$ & $\begin{array}{l}\text { Power } \\
(\mathrm{mW})\end{array}$ & $\begin{array}{c}\text { Voltage } \\
(\mathrm{mV})\end{array}$ & $\begin{array}{c}\text { Current } \\
(\mathbf{m A})\end{array}$ & $\begin{array}{r}\text { Power } \\
(\mathrm{mW})\end{array}$ \\
\hline 438.2 & 21 & 4.03 & 85.25 & 26 & 3.23 & 83.98 \\
\hline 512 & 24 & 4.06 & 89.32 & 26 & 3.47 & 90.22 \\
\hline 612 & 25 & 3.85 & 96.25 & 27 & 3.52 & 95.04 \\
\hline 724 & 28 & 3.55 & 99.4 & 25 & 3.95 & 98.75 \\
\hline
\end{tabular}

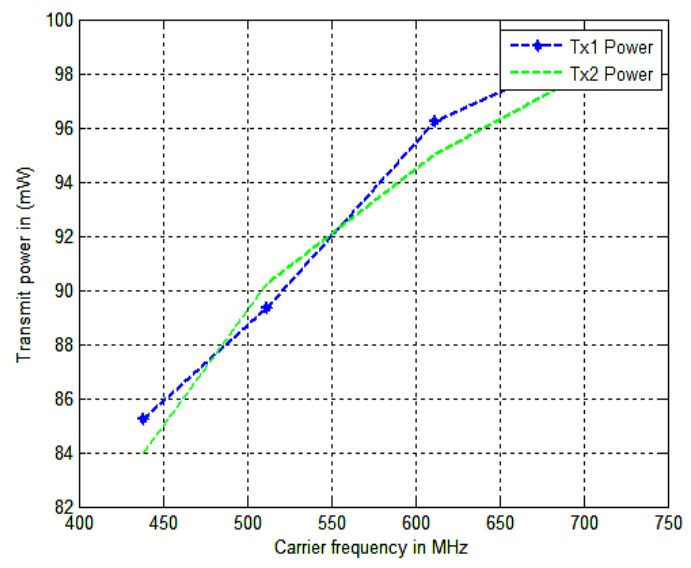

Fig.7. Observation on the basis of transmit power vs. Carrier Frequency for USRP transmitter

USRP-2922 operates in the frequency range of $400 \mathrm{MHz}$ to 4.4GHz. The transmit power for USRP is given as $50 \mathrm{~mW}$ $100 \mathrm{~mW}$. For calculation of exact power in case of analysis the transmit power of device is measured directly with oscilloscope and current and voltage probes. Here, it is observed that the circuit power of the device at transmitter side depends on transmitter circuit power and the total transmit power. Hence, in order to make system energy efficient power consumption of the system is considered. By considering reduced transmit power the required energy per bit for fixed symbol rate in case of testbed can be computed. In this paper, it is shown that carrier frequency is related with transmit power. The Fig. 6 gives the measurement setup of transmit power using oscilloscope. The Table. 3 gives the respective values of transmit power when different carrier frequencies are chosen. From Fig.7, it is observed that the lower the carrier frequency lower is the transmit power.

\section{SIMULATION RESULTS}

In this section, we discussed some other parameters which are closely related with the energy or power of the system. Evaluation of the results is carried out in LABVIEW math script. Energy efficiency and spectral efficiency tradeoff is observed for different configurations of transmit and receive antenna pairs as shown in Fig.8. The Fig.9 shows the effect of variation of symbol rate on the energy consumption per bit. As symbol rate increases bit duration decreases which in turn lowers the energy consumption of the system. In Fig.10, it is observed that when the transmission distance increases energy consumption per bit also increases. Thus, the increased energy consumption can be reduced by optimizing transmission time and bandwidth. From Fig.11, it is observed that when both circuit power and transmit power of the system is considered for fixed symbol rate then the energy consumption of the system increases.

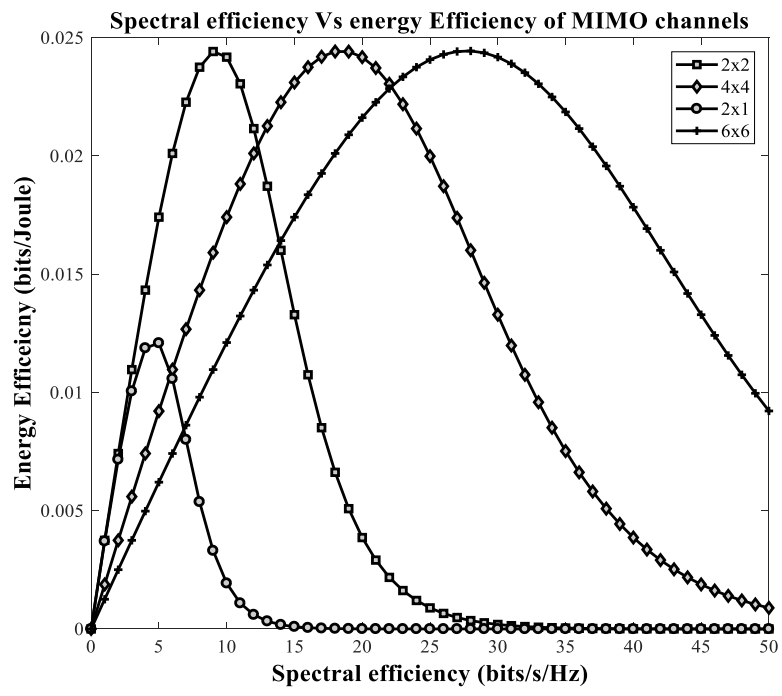

Fig.8. Energy efficiency vs. Spectral efficiency of MIMO system

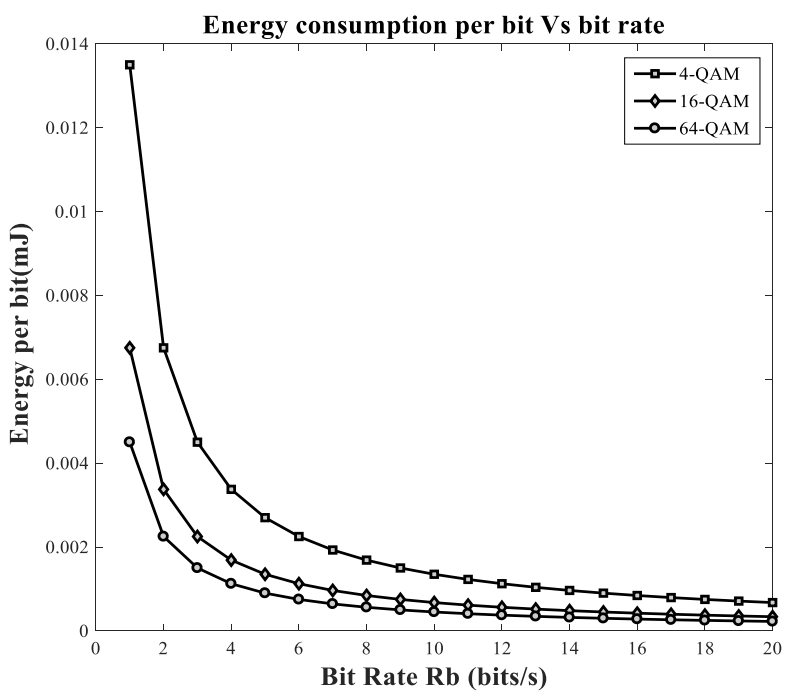

Fig.9. Energy per bit vs. Symbol Rate 


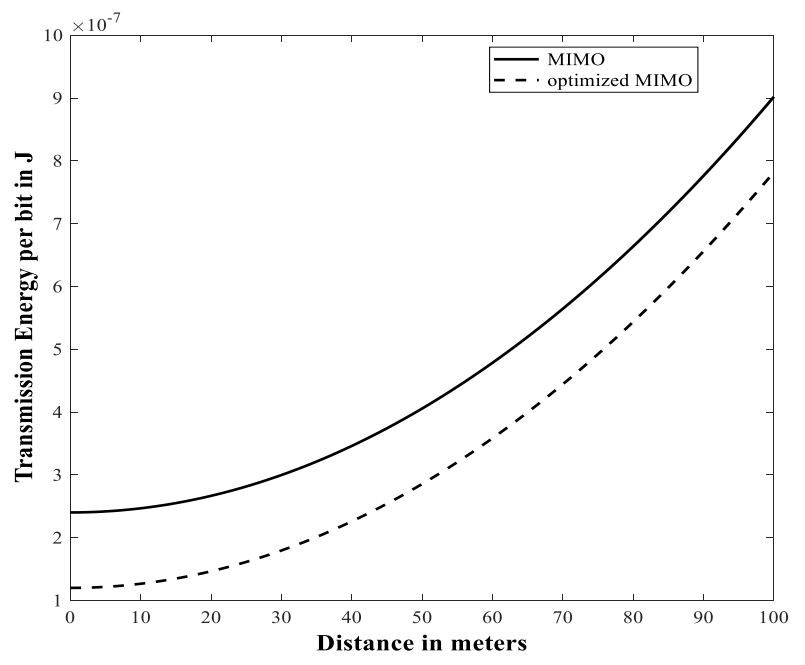

Fig.10. Transmission energy per bit vs. Distance for MIMO system

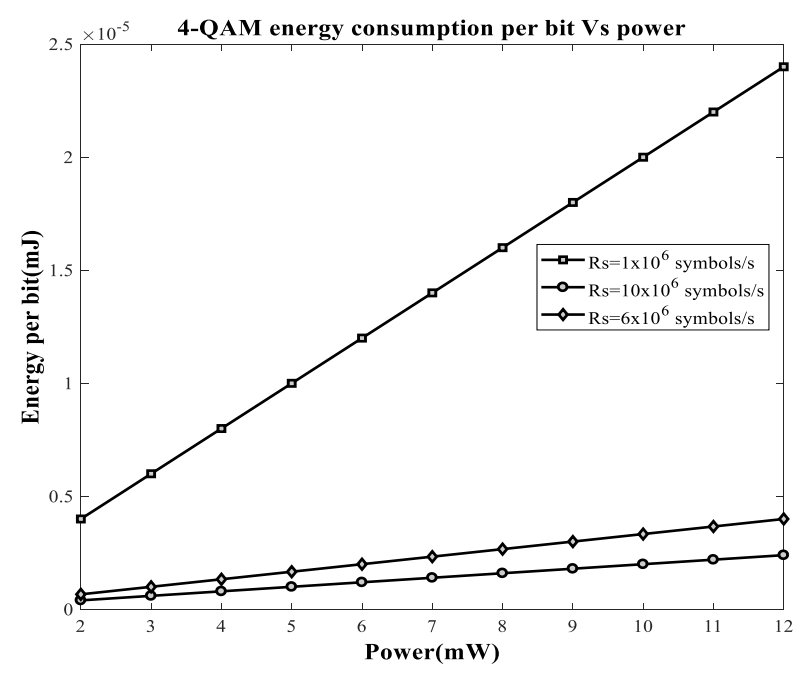

Fig.11. Energy per bit vs. Power (mW)

\section{CONCLUSION}

This paper demonstrates the implementation of $2 \times 2$ MIMO system using USRP-2922 in real world environment. QAM modulation scheme is considered \& obtained constellations is plotted for two receiver signals. Then power consumption of the RF signal chains is analyzed. This paper focuses only on transmitter energy. By allowing the testbed to measure actual transmitted power for both transmitters, the transmit power can be reduced to about $25 \%$ where, the carrier frequency is $512 \mathrm{MHz} \&$ fixed symbol rate is $1 \mathrm{Mbps}$. The performance of the system is evaluated in terms of spectral efficiency, variable symbol rate and transmission distance with help simulation results. Thus, optimizing the transmission energy leads to an energy efficient system.

\section{REFERENCES}

[1] J. Mietzner, R. Schober, L. Lampe, W.H. Gerstacker and P.A. Hoeher, "Multiple-Antenna Techniques for Wireless
Communications - A Comprehensive Literature Survey", IEEE Communications Surveys and Tutorials, Vol. 11, No. 2, pp. 87-105, 2009.

[2] G.Y. Li et al., "Energy-Efficient Wireless Communications: Tutorial, Survey, and Open Issues", IEEE Wireless Communications, Vol. 18, No. 6, pp. 28-35, 2011.

[3] L.M. Correia et al., "Challenges and Enabling Technologies for Energy Aware Mobile Radio Networks", IEEE Communications Magazine, Vol. 48, No. 11, pp. 66-72, 2010.

[4] S. Verdu, "Spectral Efficiency in the Wideband Regime", IEEE Transactions on Information Theory, Vol. 48, No. 6, pp. 1319-1343, 2002.

[5] A. El Gamal, C. Nair, B. Prabhakar, E. Uysal-Biyikoglu and S. Zahedi, "Energy-Efficient Scheduling of Packet Transmissions over Wireless Networks", Proceedings of $21^{\text {st }}$ Annual Joint Conference of the IEEE Computer and Communications Societies, Vol. 3, pp. 1773-1782, 2002.

[6] Shuguang Cui, A.J. Goldsmith and A. Bahai, "Modulation Optimization under Energy Constraints", Proceedings of IEEE International Conference on Communications, pp. 2805-2811, 2003.

[7] Shuguang Cui, A.J. Goldsmith and A. Bahai, "EnergyConstrained Modulation Optimization", IEEE Transactions on Wireless Communications, Vol. 4, No. 5, pp. 2349-2360, 2005.

[8] C. Schurgers, O. Aberthorne and M.B. Srivastava, "Modulation Scaling for Energy Aware Communication Systems", Proceedings of International Symposium on Low Power Electronics and Design, pp. 96-99, 2001.

[9] Shuguang Cui, A.J. Goldsmith and A. Bahai, "EnergyEfficiency of MIMO and Cooperative MIMO Techniques in Sensor Networks", IEEE Journal on Selected Areas in Communications, Vol. 22, No. 6, pp. 1089-1098, 2004.

[10] H. Li, L. Song, D. Zhu and M. Lei, "Energy Efficiency of Large Scale MIMO Systems with Transmit Antenna Selection", Proceedings of IEEE International Conference on Communications, pp. 4641-4645, 2013.

[11] C. Jiang and L.J. Cimini, "Antenna Selection for EnergyEfficient MIMO Transmission", IEEE Wireless Communications Letters, Vol. 1, No. 6, pp. 577-580, 2012.

[12] Huan-Chang Liu, J.S. Min and H. Samueli, "A Low-Power Baseband Receiver IC for Frequency-Hopped Spread Spectrum Communications", IEEE Journal of Solid-State Circuits, Vol. 31, No. 3, pp. 384-394, 1996.

[13] S.M. Alamouti, "A Simple Transmit Diversity Technique for Wireless Communications”, IEEE Journal on Selected Areas in Communications, Vol. 16, No. 8, pp. 1451-1458, 1998.

[14] Ben Zid Maha and Raoof Kosai, "Multi User MIMO Communication: Basic Aspects, Benefits and Challenges", Proceedings of Recent Trends in Multi-user MIMO Communications, pp. 1-22, 2013.

[15] G. Soni, G. Kaur and V.K. Banga, "Implementation and BER Analysis of 2×2 MIMO using USRP 2920- Universal Software Radio Peripheral", Proceedings of $2^{\text {nd }}$ International Conference on Computational Intelligence and Communication Technology, pp. 523-527, 2016.

[16] J.G. Prokis, "Digital Communications", $4^{\text {th }}$ Edition, McGraw-Hill, 2000. 